Cahiers Charlevoix

Études franco-ontariennes
Cahiers Charlevoix Études franco-ontariennes

or Crevenerix of

\title{
Les Médias en Ontario chez les francophones et les anglophones
}

\section{Simon Laflamme}

Volume 6, 2005

URI : https://id.erudit.org/iderudit/1039334ar

DOI : https://doi.org/10.7202/1039334ar

Aller au sommaire du numéro

\section{Éditeur(s)}

Société Charlevoix

Presses de l’Université d’Ottawa

\section{ISSN}

1203-4371 (imprimé)

2371-6878 (numérique)

Découvrir la revue

Citer cet article

Laflamme, S. (2005). Les Médias en Ontario chez les francophones et les anglophones. Cahiers Charlevoix, 6, 249-282. https://doi.org/10.7202/1039334ar
Résumé de l'article

L'étude que Simon Laflamme livre ici est tirée d'une enquête menée à Sudbury en 2001 et en 2002 auprès de plusieurs centaines de personnes pour connaître la place des médias et la fréquence de leur utilisation dans les familles franco-ontariennes en comparaison des familles anglophones. À l'exception du domaine de l'informatique (ordinateur, courriel et toile électronique), il note peu de distinctions entre les deux groupes culturels en ce qui a trait à la possession et à l'usage des appareils et moyens de communication (téléviseur et magnétoscope, téléphone et télécopieur, lecteur de disques, radio et chaîne audio, livre, correspondance), car tous les deux s'inscrivent dans un même "processus simultané d'homogénéisation et d'hétérogénéisation " propre à la société postmoderne. Il note toutefois que l'âge et l'instruction ont une incidence sur l'usage des nouvelles technologies plus marquée même que la langue des usagers. Son analyse ouvre ainsi la porte sur un autre volet qui envisagerait les contenus véhiculés, les messages produits et consommés.
Ce document est protégé par la loi sur le droit d'auteur. L'utilisation des services d’Érudit (y compris la reproduction) est assujettie à sa politique d'utilisation que vous pouvez consulter en ligne.

https://apropos.erudit.org/fr/usagers/politique-dutilisation/ 


\title{
Les Médias en Ontario
}

\section{CHEZ LES FRANCOPHONES}

\section{ET LES ANGLOPHONES}

\author{
Simon Laflamme
}

Département de sociologie Université Laurentienne 
SOMMAIRE

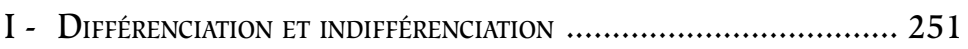

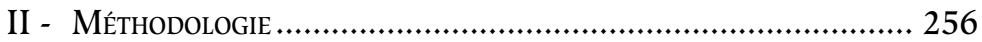

III - Possession et USAGE DeS MÉdias ……….................................... 257

A. Les médias dont on dispose au foyer ............................. 257

B. L'usage des médias .......................................................... 259

1. La télévision et les vidéos

2. Le téléphone

3. La radio

4. La musique enregistrée

5. La lecture

6. La correspondance

7. L'informatique

IV - INDIFFÉRENCIATION ET DIFFÉRENCIATION

268

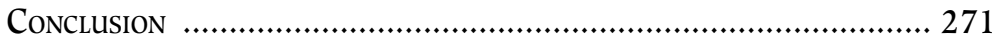

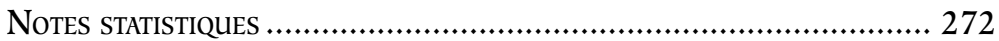

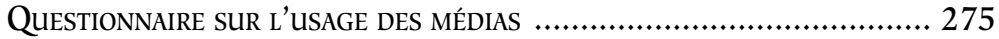




\section{Les Médias en OntaRio}

\section{CHEZ LES FRANCOPHONES}

\section{ET LES ANGLOPHONES}

\section{DiffÉrenciation et indifférenciation}

Les questions relatives aux technologies de communication occupent de plus en plus d'espace dans le champ des sciences sociales. Cela se comprend facilement puisque, d'une part, aux cours des deux dernières décennies, ces technologies ont connu une évolution rapide associée à un usage croissant ${ }^{1}$ et que, d'autre part, les sociétés sont fortement conditionnées par la manière dont circule en elles l'information, cette circulation étant en partie ellemême déterminée par les moyens de communication ${ }^{2}$. Cette dialectique des sociétés et des médias contribue largement à la définition d'une culture. Or, dans la mesure où cette dialectique dépend de la nature des médias, on peut s'attendre à des variations selon l'évolution médiatique qu'on

\footnotetext{
${ }^{1}$ Lire, par exemple, Manuel Castells, The Information Age: Economy, Society and Culture, Oxford, Blackwell, vol. I, 1996, vol. 2, 1997, vol. 3, 1998.

${ }^{2}$ Position théorique qui prend diverses configurations, de Marshall McLuhan (La Galaxie de Gutemberg. La Genèse de l'homme typographique, Montréal, HMH, 1968 [1962]; Pour comprendre les médias, Paris, Seuil, 1968 [1964]) à Jean Baudrillard (La Société de consommation, ses mythes, ses structures, Paris, Denoël, 1970; Le Paroxyste indifférent. Entretiens avec Philippe Petit, Paris, Grasset, 1997).
} 
observe dans une communauté donnée, et l'on sait que cette évolution dépend fortement de facteurs sociodémographiques comme le revenu ou le niveau d'instruction $^{3}$. Ainsi, dans une communauté plus riche, on devrait trouver une structure médiatique mieux développée que dans une communauté plus pauvre, de même que, dans une collectivité plus instruite, des usages différents de ceux d'une collectivité moins instruite 4 . Ces variations sont lourdes de conséquences. Elles font que les communautés les moins riches et les moins instruites entretiennent forcément un rapport différencié à l'information - à celle qu'elles reçoivent et à celle qu'elles produisent -, et les méfaits de cette différenciation sont d'autant plus importants que les médias auxquels une communauté a moins accès contribuent à l'institutionnalisation de l'information - à la fabrication des discours de référence - ou permettent d'inscrire les agents communicants dans l'univers de la circulation de l'information. Il est donc important de bien comprendre quels sont les médias dont dispose une collectivité et de quelle manière elle y recourt.

Dans le cas des rapports entre minoritaire et majoritaire, cette compréhension est d'autant plus importante que le groupe majoritaire participe de l'ensemble des informations institutionnalisables. Un groupe majoritaire qui est peu en mesure de faire circuler des messages qui seront relayés par l'ensemble des moyens de communication de masse, ou dont les usages médiatiques sont peu fréquents et sont réduits à des médias peu nombreux ou peu développés, n'aura pas, dans la contemporanéité, d'influence

\footnotetext{
${ }^{3}$ Pour ce qui est du rapport à l'informatique, on lira les données d'Ann Denis et Michèle Ollivier dans «L'Écart numérique au Canada. L'intersection du genre, du statut socio-économique et de la langue d'usage» (communication présentée au Colloque du Réseau des chercheures féministes de l'Ontario français, Toronto, avril 2002). Ces données montrent bien que la probabilité d'avoir chez soi le média de même que l'usage de ce média croissent avec la richesse et l'instruction.

${ }^{4}$ Voir, par exemple, le site de Nua Internet Surveys, http://www.nua.ie/surveys/.
} 
significative et durable sur la culture du groupe minoritaire. Un groupe majoritaire, cependant, dont la langue est celle qui livre le plus d'informations à l'ensemble des messages médiatiques, affectera nécessairement le groupe minoritaire qui parle une autre langue; et cette empreinte sera d'autant plus profonde que l'écart qui s'établira à la faveur du majoritaire sera grand pour ce qui est des usages des divers moyens de communication ${ }^{5}$.

Prenant en considération l'influence de ces univers communicationnels, les sciences sociales comprennent le devenir des sociétés contemporaines de deux façons: ces sociétés tendent ou bien à s'homogénéiser ou bien à s'hétérogénéiser. La tendance à l'indifférenciation a d'abord été perçue par évocation de l'influence des moyens de communication de masse. L'école de Francfort affirmait que la logique capitaliste à laquelle répondait l'industrie médiatique faisait en sorte que les messages perdaient en rationalité, atteignaient des ensembles de plus en plus vastes de destinataires qui devenaient ainsi de plus en plus semblables ${ }^{6}$. La théorie des industries culturelles ira exactement dans le même sens ${ }^{7}$. Tiendra également un propos de cet ordre la théorie de la cultivation ${ }^{8}$. La thèse

\footnotetext{
${ }^{5}$ Simon Laflamme, «Les Médias en milieu minoritaire: les rapports entre l'économie et la culture", in Fernand Harvey (dir.), Médias francophones hors Québec et identité, analyses, essais et témoignages, Québec, Institut québécois de recherche sur la culture, 1992, p. 25-35.

6 Theodor W.-Adorno, Modèles critiques, Paris, Payot, 1984 [1963]. Max Horkheimer et Theodor W.- Adorno, La Dialectique de la raison. Fragments philosophiques, Paris, Gallimard, Bibliothèque des idées, 1974 [1944].

${ }^{7}$ Jean-Guy Lacroix, «Présentation: Pour une théorie des industries culturelles ", Cahiers de recherche sociologique, vol. 4, n 2, automne 1986, p. 5-18. Armand Mattelart, Multinationales et systèmes de communication, Paris, Anthropos, 1976. Bernard Miège, La Société conquise par la communication, Grenoble, Presses de l'Université de Grenoble, coll. «Logiques sociales», 1996.

${ }^{8}$ George Gerbner, "Mass Media and Human Communication", in F. E. X. Dance (dir.), Human Communication Theory, New York, Holt, Rinehart, 1967, p. 40-57.
} 
sera reprise, quoique dans un contexte théorique plus général, dans Le Grand Système de Georges Balandier:

La surmodernité mondialisante paraît recouvrir, courant envahissant et en apparence irrésistible, tout ce qui est constitué dans la diversité. Elle met celle-ci en péril de rapide érosion. Elle a la puissance acquise pour moteur, la technique et le marché pour énergie, le capitalisme libéral pour orientation de ses flux. Un monde s'engendre qui asservit les systèmes symboliques à mesure de la montée en puissance du faire transformateur?.

La tendance à la différenciation, elle, trouve ses origines dans la phénoménologie où l'on procède à une lecture individualisante du social, à une lecture qui donne essentiellement à voir des personnes, des sujets qui apparaissent de plus en plus distincts les uns des autres. La multiplicité, la variation des sources d'information produisent des personnes qui trouvent de moins en moins dans la tradition un discours qui corresponde à leur être. L'univers se renouvelle constamment et produit ainsi la diversité ${ }^{10}$. Dans ce développement postmoderne ${ }^{11}$, la liberté est telle que la socialité même est menacée ${ }^{12}$.

À la lumière de ces notions théoriques, on peut s'interroger sur la situation des francophones en Ontario. Cette interrogation est importante, ne serait-ce que parce qu'elle permettra de comprendre la situation d'une population qui, tout en étant minoritaire sur le plan linguistique, a constitué un terrain propice pour l'implantation des nouvelles technologies de communication, notamment à cause du niveau de richesse relativement élevé qu'on observe au

\footnotetext{
${ }^{9}$ Georges Balandier, Le Grand Système, Paris, Fayard, 2001, p. 18-19.

${ }^{10}$ Yves Boisvert, Le Postmodernisme, Montréal, Boréal, 1995.

${ }^{11}$ La postmodernité caractérise les sociétés postindustrielles, c'est-à-dire celles dans lesquelles le secteur tertiaire est grandement développé, souvent plus développé que le secteur secondaire. Dans ces sociétés, les médias de masse jouent normalement un grand rôle et ces médias connaissent une forte concurrence.

${ }^{12}$ Alain Touraine, Pourrons-nous vivre ensemble? Égaux et différents, Paris, Fayard, 1997.
} 
Canada et particulièrement en Ontario. Elle l'est également parce qu'elle oblige à comparer cette situation à celle d'une population majoritaire qui parle la langue la plus commune dans l'univers des messages de masse - l'anglais. Elle l'est à un troisième chef parce qu'elle servira à observer la manière dont se présentent les pratiques médiatiques dans une société postindustrialisée.

De récents travaux ont établi que la population francoontarienne connaît peu de discrimination selon le revenu quand on lui donne pour point de comparaison la population anglo-ontarienne. La structure de ses professions, toutefois, n'est pas la même que celle des anglophones de la province: on y trouve, par exemple, en proportion, davantage de travailleurs du secteur primaire et moins de cadres. En outre, d'une façon générale, les Franco-Ontariens s'avèrent moins instruits que les Anglo-Ontariens ${ }^{13}$. La situation est donc quelque peu confuse. On devrait s'attendre à découvrir chez les francophones et chez les anglophones très peu de différences quant à la possession des divers médias, mais certains écarts quant à la fréquence des usages des nouvelles technologies à l'avantage des anglophones. Si, par ailleurs, le jeune âge n'atténue pas ces divergences, cela signifiera qu'elles sont bel et bien inscrites dans le devenir des deux populations. On notera qu'une étude préliminaire d'Ann Denis et de Michèle Ollivier laisse entrevoir très peu de transformations quant à la différence entre francophones et anglophones; mais elle livre aussi

\footnotetext{
${ }^{13}$ Anne Gilbert et André Langlois, Regard sur les nouvelles réalités francoontariennes, Ottawa, Association canadienne-française de l'Ontario (ACFO), 1994. Christiane Bernier et Simon Laflamme, "Discrimination sexuelle et discrimination linguistique: lecture des inégalités salariales au Canada et en Ontario», Revue du Nouvel-Ontario, no 27, 2002, p. 63-91. Diane Farmer, Artisans de la modernité. Les Centres culturels en Ontario français, Ottawa, Les Presses de l'Université d'Ottawa, 1996. Anne Gilbert, Espaces francoontariens, Ottawa, Le Nordir, 1998. Office des affaires francophones, Profil statistique. Les francophones en Ontario, Toronto, Gouvernement de l'Ontario, août 1999.
} 
des résultats ambigus - peut-être à l'image de la complexité de la situation - que la petite taille de l'échantillon et la définition de l'instrument de collecte de données ne permettent pas d'explorer ${ }^{14}$.

La question de savoir si la postmodernité donne lieu à la différenciation ou à l'homogénéité est lourde de sens. Il nous semble toutefois qu'on n'ait pas vraiment affaire à une alternative, que les deux tendances caractérisent les nouvelles sociétés, celles dans lesquelles les moyens de communication donnent lieu à des usages extensifs et intensifs. Si cette hypothèse est juste, les analyses devraient donner à observer aussi bien des phénomènes récurrents que des variations individuelles.

\section{Méthodologie}

Pour effectuer les analyses grâce auxquelles on pourra connaître la situation franco-ontarienne quant à l'usage des médias, la comparer à celle des Anglo-Ontariens et vérifier les hypothèses sur les rapports entre minorité et majorité aussi bien que sur les tendances de la société postmoderne, une enquête a été effectuée. Un questionnaire a été conçu en anglais et en français ${ }^{15}$. Il a pour fin de découvrir quels médias et quels services médiatiques on trouve dans le foyer du répondant de même que les usages qu'en fait ce répondant. Il a été distribué dans la région de Sudbury à deux moments. Au printemps de l'année 2001, les responsables de la collecte de données ont distribué la version française. S'est vite manifestée la nécessité de comparer les résultats obtenus à ceux de la population anglophone. Une deuxième collecte a alors eu lieu, au printemps 2002, ciblant, cette fois, la population anglophone. L'échantillon est donc stratifié selon la langue. Lors de la première

\footnotetext{
${ }^{14}$ Op. cit.

${ }^{15}$ Ce document, que nous avons développé en français, apparaît en annexe. La version anglaise est la traduction de la française.
} 
collecte, un peu plus d'une trentaine d'individus ont fait circuler le questionnaire dans leur environnement et en ont rapporté environ une vingtaine chacun, soit $703 \mathrm{au}$ total. La deuxième fois, les responsables de la collecte étaient au nombre de treize et leur tâche était la même; l'objectif était alors de recueillir au moins 200 questionnaires afin de rendre possible certaines analyses multivariées; l'objectif a été atteint puisque 252 questionnaires ont été obtenus ${ }^{16}$.

La proportion d'hommes et de femmes n'est pas statistiquement différente d'un échantillon à l'autre(1); toutefois, dans les deux cas, les femmes représentent une proportion légèrement supérieure $(62,2 \%$ chez les francophones et $55,6 \%$ chez les anglophones) à celle des hommes. Dans l'ensemble de l'échantillon, les personnes sont âgées de 15 à 92 ans. L'âge moyen est inégal pour les deux groupes linguistiques: les individus qui composent l'échantillon anglophone sont, en moyenne, plus vieux que ceux qui appartiennent à l'échantillon francophone ${ }^{(2)}$; cela implique qu'il faudra privilégier les analyses où l'âge est traité comme variable nominale, et non comme variable cardinale, c'està-dire où les comparaisons se font par tranches d'âge.

\section{Possession et usage des médias}

\section{A. LES MÉDIAS DONT ON DISPOSE AU FOYER}

Le questionnaire comportait dix-neuf questions sur les médias du foyer ou, parfois, sur la possibilité de disposer d'un média. Si l'on distribue les réponses selon les deux échantillons dans leur ensemble, on observe des proportions égales chez les francophones et les anglophones pour

\footnotetext{
${ }^{16}$ Les notes qui comportent les informations statistiques relatives aux tests effectués se trouvent en fin de document. Pour distinguer ce système de renvoi de celui qui se rapporte aux notes infrapaginales, la numérotation dans le texte est mise entre parenthèses. L'exposant ${ }^{(1)}$, par exemple, indique que la note $« 1 »$ se trouve à la fin du texte.
} 
six médias - ou services médiatiques - : le fait d'avoir chez soi un téléviseur à écran géant ${ }^{(3)}$, des appareils radio ${ }^{(4)}$, un télécopieur ${ }^{(5)}$, un afficheur sur la ligne téléphonique ${ }^{(6)}$, un téléphone cellulaire ${ }^{(7)}$ et un scanner ${ }^{(8)}$. Les anglophones ont un avantage pour neuf médias: le magnétoscope ${ }^{(9)}$, le lecteur DVD ${ }^{(10)}$, le lecteur de disques compacts ${ }^{(11)}$, le fait de disposer d'un télécopieur ${ }^{(12)}$, le portable pour le téléphone du réseau fixe ${ }^{(13)}$, le répondeur sur le téléphone cellulaire ${ }^{(14)}$, l'ordinateur ${ }^{(15)}$, le branchement Internet ${ }^{(16)}$ et le service de courrier électronique ${ }^{(17)}$. Les proportions sont plus élevées chez les francophones pour quatre médias : le téléviseur ${ }^{(18)}$, le service du câble ou d'une antenne parabolique ${ }^{(19)}$, la ligne téléphonique ${ }^{(20)}$ et le branchement à un répondeur $\mathrm{du}$ téléphone sur le réseau fixe ${ }^{(21)}$. Dans les cas d'inégalité entre les deux groupes, les différences de proportion sont inférieures à $10 \%$, sauf pour ce qui est de l'informatique. À la question «Dans votre domicile, y a-t-il un ordinateur?", par exemple, la réponse est affirmative pour $86,9 \%$ des anglophones, mais seulement pour $65,9 \%$ des francophones; les chiffres relatifs à Internet et au courriel sont semblables ${ }^{17}$. Si l'on effectue les mêmes analyses, mais en ne les faisant porter, cette fois, que sur les personnes qui ont 35 ans ou moins et qui travaillent à plein temps ou à temps partiel, le nombre des égalités de proportion augmente: il passe de six à douze. Les inégalités subsistent ou surgissent pour sept médias; elles sont à l'avantage des francophones dans quatre cas: le téléviseur ${ }^{(2)}$, le téléviseur à écran géant ${ }^{(23)}$, le service du câble ou le branchement à une antenne parabolique ${ }^{(24)}$ et le répondeur pour le téléphone du réseau fixe; dans les trois autres cas, elles favorisent les anglophones, soit le lecteur $\mathrm{DVD}^{(25)}$, le répondeur pour le téléphone cellulaire ${ }^{(26)}$ et l'ordinateur ${ }^{(27)}$.

\footnotetext{
${ }^{17}$ Denis et Ollivier ont, elles aussi, observé un avantage pour les anglophones ( L'Écart numérique au Canada. L'intersection du genre, du statut socioéconomique et de la langue d'usage», op. cit.).
} 
Les différences sont généralement faibles, sauf pour l'ordinateur du foyer où elle est de $22,4 \%$, toujours en faveur des anglophones. Ainsi, les différences sont moins fréquentes si l'on ne prend que les jeunes qui sont sur le marché du travail, mais les francophones restent moins nombreux à faire usage d'un ordinateur au sein du foyer. Le niveau de scolarité, toutefois, élimine cet écart pour ce qui est du fait de disposer d'un ordinateur au foyer. En effet, si l'on prend les jeunes de 35 ans ou moins qui sont sur le marché du travail et qui ont fait des études supérieures, l'analyse révèle un cas d'égalitéé( ${ }^{(2)}$; il en va pareillement si l'on prend tous les jeunes du même groupe d'âge pour le même niveau d'instruction ${ }^{(29)}$. Dans l'ensemble des personnes qui ont obtenu un diplôme de premier cycle universitaire, on ne détecte pas non plus de différence entre francophones et anglophones, qu'il s'agisse de tous les jeunes de 35 ans ou moins ${ }^{(30)}$ ou seulement de ceux qui occupent un emploi ${ }^{(31)}$. Le fait, donc, que les francophones tendent moins que les anglophones à munir leur foyer d'un ordinateur est largement attribuable au fait qu'ils sont, dans l'ensemble, moins instruits.

\section{B. L'USAGE DES MÉDIAS}

La totalité des variables qui portent sur l'usage des médias sont d'ordre cardinal; elles peuvent donc donner lieu à des comparaisons de moyennes. Ces comparaisons sont toutes effectuées à partir d'analyses de variance impliquant toutes au moins trois facteurs: le niveau de scolarité, dichotomisé selon que les personnes ont atteint le niveau des études postsecondaires ou non; l'âge, divisé en quatre catégories que sont 15 à 25 ans, 26 à 35 ans, 36 à 50 ans et 51 ans ou plus, et, bien entendu, la langue du questionnaire. Il est apparu utile de distinguer les personnes qui ont atteint le niveau des études postsecondaires des autres puisqu'il est plus probable qu'elles soient exposées aux nouvelles technologies; en outre, les sciences 
sociales ont maintes fois montré que les comportements des moins instruits diffèrent de ceux des plus instruits. Il est aussi apparu important de différencier les âges, car on sait que les plus jeunes sont plus susceptibles d'être exposés aux nouvelles technologies, de même qu'il est bien connu que leurs habitudes à l'égard des médias ne sont pas forcément semblables à celles des plus vieux. En marge de ces analyses, l'occupation a été prise en considération, à savoir si les personnes sont ou non sur le marché du travail; cette variable n'a rien révélé d'essentiel qui ne transparaisse déjà dans l'âge et le niveau d'instruction; il en est de même pour les analyses faites à partir des occupations classifiées dans une échelle cardinale telle que l'ont proposée Blishen, Carroll et Moore ${ }^{18}$ - cette échelle manquant sans doute de sensibilité pour une analyse comme celle qui est ici réalisée. Le revenu n'a pas été mesuré en lui-même dans le questionnaire; quoi qu'il en soit, s'il a certainement une incidence sur la possession et sur l'usage d'équipement, il ne faut pas oublier que les Franco-Ontariens ne constituent pas une communauté plus pauvre que ne le sont les AngloOntariens; il faut bien comprendre que la variable la plus importante, ici, c'est le niveau de scolarité, et que ce qui distingue le plus les Franco-Ontariens des autres Ontariens, c'est leur difficulté à atteindre le niveau des études postsecondaires, et cela depuis longtemps ${ }^{19}$.

Les analyses se concentreront maintenant sur chacun des registres médiatiques.

\footnotetext{
${ }^{18}$ Bernard R. Blishen, William K. Carroll et Catherine Moore, «The 1981 socioeconomic index for occupations in Canada", Revue canadienne de sociologie et d'anthropologie, vol. 24, no 4, 1987, p. 465-488.

${ }^{19}$ Stacy Churchill, Saeed Quazi et Normand Frenette, Éducation et besoins des Franco-Ontariens: le diagnostic d'un système d'éducation, vol. I et II, Toronto, Conseil de l'éducation franco-ontarienne, 1985.
} 


\section{La télévision et les vidéos}

Si l'on compare, dans l'ensemble, les francophones et les anglophones, on ne note pas de différence pour ce qui est du temps consacré à regarder la télévision: les premiers passent 15,67 heures par semaine devant leur écran, les seconds $15,89^{(32)}$. Si l'on ne prend que les jeunes de 35 ans ou moins, qu'il s'agisse de la totalité d'entre eux ${ }^{(33)}$ ou strictement de ceux qui occupent un emploi ${ }^{(34)}$, les moyennes restent égales.

Le fait de visionner des films vidéos mensuellement (VHS ou DVD) constitue aussi une activité qui ne connaît pas de distinction selon la langue. Il est cependant plus habituel chez les plus jeunes que chez les plus vieux. Les jeunes qui travaillent regardent, en moyenne, entre six et neuf vidéos par semaine, qu'ils soient anglophones ou francophones ${ }^{(35)}$; l'ensemble de tous les jeunes, lui, entre six et sept vidéos, toujours sans que la langue du répondant fasse varier cette statistique $^{(36)}$.

\section{Le téléphone}

Pour évaluer l'usage du téléphone, le questionnaire fournissait d'abord quatre sources de données : le nombre d'appels téléphoniques locaux et le nombre d'appels interurbains, dans le cadre du travail et en dehors de ce cadre, sur une base hebdomadaire. Les moyennes qui sont calculées ne connaissent pas de variation selon quelque facteur que ce soit. Cette indétermination des facteurs est parfois attribuable au fait que les moyennes sont très proches les unes des autres, comme c'est le cas relativement au niveau d'instruction où, en ce qui a trait aux communications téléphoniques reliées au travail, les personnes qui ont fait des études postsecondaires présentent une moyenne de 33,69 appels téléphoniques et les autres, de 30,66. Mais l'indétermination s'explique aussi par le fait que les différences individuelles sont souvent plus 
importantes que celles qui pourraient être reliées aux facteurs eux-mêmes: c'est ainsi, par exemple, que les variations selon l'âge passent de 22,97 communications locales associées au travail, par semaine, chez les individus de 25 ans ou moins à 36,11 chez ceux qui ont entre 36 et 50 ans, sans que cet écart soit considéré comme significatif, tout simplement parce que les écarts types de ces deux moyennes sont respectivement de 43,37 et de 76,14.

Le questionnaire offrait, ensuite, une autre source d'information. Les répondants, en effet, étaient invités à estimer le nombre d'heures par semaine au cours desquelles ils communiquaient par téléphone à l'extérieur du cadre de leur travail. L'âge s'est ici révélé déterminant ${ }^{(37)}$ : les plus jeunes et les plus vieux sont ceux qui passent le plus de temps au téléphone (respectivement 8,53 et 7,29 heures par semaine); les personnes qui ont entre 26 et 35 ans et celles qui ont entre 36 et 50 ans affichent des chiffres un peu plus faibles, soit 5,61 et 5,20 heures par semaine.

\section{La radio}

L'instrument de collecte de données comportait une question sur l'exposition à la radio; les personnes interrogées devaient estimer le temps hebdomadaire durant lequel elles l'écoutaient. L'analyse de variance n'a repéré ni effet principal, ni effet d'interaction. Les moyennes sont très semblables - environ onze heures par semaine -; mais les écarts types sont aussi élevés. Cela signifie que les phénomènes sont assez semblables d'une catégorie sociale à l'autre, mais que les différences entre les individus sont importantes.

\section{La musique enregistrée}

Le temps d'écoute de la musique enregistrée ne différencie pas les anglophones des francophones ${ }^{(38)}$. Il distingue 
cependant les plus jeunes des autres ${ }^{(39)}$; ils y consacrent, en effet, 14,15 heures par semaine en moyenne, alors que les trois autres groupes d'âge ne réservent à cette activité que de 4,28 à 5,84 heures par semaine ${ }^{20}$.

\section{La lecture}

La lecture peut être étudiée de diverses façons. Le temps qui y est réservé en est un bon indicateur. Pour cette raison, le questionnaire a demandé combien de temps on lui consacrait en dehors des études et du travail. La langue ne s'est pas révélée un facteur déterminant ${ }^{(40)}$, contrairement à l'âge et à l'instruction. L'effet de l'âge est simple: plus le groupe est vieux, plus la moyenne du temps de lecture est élevée - les personnes qui ont plus de 50 ans lisent 8,02 heures par semaine, celles qui ont entre 36 et 50 ans, 5,18, celles qui ont entre 26 et 35 ans, 4,91, les plus jeunes, $4,26^{(41)}$. L'effet de la scolarisation n'est pas moins évident: avec l'instruction s'accroît l'inclination à lire ${ }^{(42)}$, et si l'on recourt à une échelle qui comporte plusieurs niveaux de scolarité, par exemple de «quelques années du cours élémentaire " à "diplôme d'études supérieures", la tendance ne s'altère jamais. Sont aussi de bons indicateurs de l'activité qu'est la lecture les rapports aux quotidiens, aux magazines et aux bibliothèques. Par conséquent, le questionnaire a demandé aux personnes qui le remplissaient d'évaluer, sur une échelle de Likert à cinq niveaux, la manière dont elles s'adonnaient à ces activités, la valeur « 1 » signifiant «jamais », la valeur « 5 » signifiant "souvent», les autres valeurs représentant des attitudes intermédiaires. Les réactions à l'énoncé «Je lis des quotidiens imprimés » rappellent le même effet d'âge ${ }^{(43)}$ : avec l'âge s'accroît l'inclination à la lecture, la moyenne

\footnotetext{
${ }^{20}$ Cet effet de l'âge sur l'écoute de la musique enregistrée a déjà été observé dans Vivre dans l'alternance linguistique. Médias, langue et littératie en Ontario français (Simon Laflamme et Christiane Bernier, Centre FORA, 1998).
} 
passant de 2,65 chez les plus jeunes à 3,77 chez les plus vieux. Elles rappellent également l'effet de l'instruction ${ }^{(44)}$. Elles révèlent, par ailleurs, un effet de la langue ${ }^{(45)}$, mais qui est très faible, se rapportant à des moyennes de 3,24 pour les francophones et de 3,13 pour les anglophones. Les positions relatives à l'énoncé «Je lis des revues ou des magazines imprimés » confirment l'influence du niveau de scolaritée $^{(46)}$, mais pas celle de l'âge ${ }^{(47)}$. La lecture des magazines est uniforme à travers l'âge, elle oscille entre 3,08 et 3,20 - ce qui, par ailleurs, ne préjuge pas des contenus de ces lectures qui sont très certainement très variés. La langue se fait à nouveau déterminante ${ }^{(48)}$, mais dans le sens inverse de ce qui a été observé pour les quotidiens; cette fois, la moyenne la plus élevée est celle des anglophones $(3,38$ contre 3,06$)$, quoique, encore une fois, la différence soit faible. L'analyse de l'activité qui consiste à fréquenter la bibliothèque, enfin, confirme l'influence de l'instruction $^{(49)}$, les plus instruits y allant plus fréquemment que les autres $(2,53$ contre 1,76$)$; elle met en évidence un effet d'âge ${ }^{(50)}$ qui va dans le sens opposé de ce qui a été constaté: si les plus vieux accordent plus de temps à la lecture et affectionnent davantage les quotidiens, ce sont les plus jeunes qui vont le plus fréquemment à la bibliothèque, les moyennes décroissant de 2,75 chez les individus qui ont entre 15 et 25 ans à 1,74 chez ceux qui ont plus de 50 ans. La langue n'a ici aucune influence ${ }^{(51)}$.

\section{La correspondance}

Quatre énoncés se rapportent au courrier traditionnel: "J'écris des lettres dans le cadre de mon travail», "J'écris des lettres en dehors du cadre de mon travail», "Je reçois des lettres dans le cadre de mon travail» et «Je reçois des lettres en dehors du cadre de mon travail». Les répondants encerclent une valeur de « 1 » à « 5 " pour chacun de ces énoncés où « 1 » signifie «jamais » et « 5 », « souvent». Dans 
les quatre cas, on trouve une influence prévisible du niveau d'instruction qui veut que les personnes qui ont fait des études postsecondaires aient des moyennes supérieures ${ }^{(52)}$. Lâge n'a d'incidence que pour deux des énoncés, soit la rédaction et la réception de lettres dans le cadre du travail $^{(53)}$, et les phénomènes sont plus récurrents chez les gens qui ont entre 26 et 50 ans que chez les plus jeunes et les plus vieux. La langue ne détermine aucunement ce rapport aux lettres ${ }^{(54)}$. Il faut noter que les moyennes sont plus élevées pour la rédaction des lettres que pour leur réception, mais qu'elles sont généralement faibles: elles franchissent difficilement la valeur de 2,0 pour la rédaction et la valeur de 2,5 pour la réception.

\section{L'informatique}

L'analyse des usages liés à l'informatique porte sur neuf variables. La première d'entre elles est le temps que les individus interrogés passent devant un écran d'ordinateur. Ont ici une influence la scolarisation ${ }^{(55)}$ et l'âge ${ }^{(56)}$, mais pas la langue ${ }^{(57)}$. La différence de moyennes entre les francophones et les anglophones est pourtant assez importante, les premiers consacrant 10,81 heures par semaine à leur ordinateur, les seconds, 15,12; la «non-inférabilité» de cette différence provient du fait que les individus présentent des temps très variés à l'intérieur de chacun de ces ensembles, comme en témoignent les écarts types de 13,33 et de 18,07, respectivement. Les variations selon l'âge sont prévisibles par certains aspects et surprenantes par d'autres: la moyenne de temps la plus élevée est celle des plus jeunes - ceux qui ont entre 15 et 25 ans -, elle est de 15,04 heures par semaine; la moyenne qui vient en second, cependant, n'est pas celle des personnes qui ont entre 26 et 35 ans $(11,33)$ comme on aurait pu le croire, elle appartient plutôt aux individus âgés de 36 à 50 ans et elle est de 14,01; les plus vieux présentent la moyenne la 
plus faible, soit 5,06. Pour ce qui est de l'influence de l'instruction, comme il fallait s'y attendre, la moyenne est plus élevée chez les plus instruits $(14,26)$ que chez les autres $(7,72)$.

Quatre autres variables ont trait au courriel. Les répondants doivent indiquer combien de messages ils écrivent et reçoivent dans le cadre de leur travail et en dehors de ce cadre. Les moyennes sont discriminées en fonction de la scolarité dans tous les cas, sauf pour les messages électroniques qui sont rédigés en dehors du cadre du travail ${ }^{(58)}$. La tendance veut néanmoins que plus on est instruit, plus on écrit de courriels et plus on en reçoit. S'il y a une exception, c'est que les différences individuelles sont, là, immenses. En effet, les personnes qui ont fait des études postsecondaires écrivent, en moyenne, deux fois plus de courriels que les autres en dehors du cadre de leur travail $(6,14$ par semaine contre 3,06$)$, mais les écarts types sont très élevés $(8,71$ et 13,92$)$, ce qui rend la différence non significative; les variations entre les individus ont donc plus d'importance que celles qui se révèlent entre les groupes. L'âge ne fait varier les moyennes que pour ce qui est des messages électroniques reçus en dehors du cadre du travail: les moyennes passent alors de 22,30 messages par semaine chez les plus jeunes à 6,07, chez les plus vieux ${ }^{(59)}$. Dans les autres cas, les différences individuelles sont trop importantes pour que les écarts soient attribuables à l'âge comme tel. La langue ne modifie aucunement les résultats, soit que les moyennes $(\bar{x})$ soient proches les unes des autres, comme c'est le cas pour les courriels écrits en dehors du cadre du travail $(4,93$ pour les francophones et 5,40 pour les anglophones) ${ }^{(60)}$, soit que les écarts types (s) soient trop imposants, comme c'est le cas pour les messages écrits dans le cadre du travail $(\bar{x}=6,58$ pour les francophones et $\overline{\mathrm{x}}=3,06$ pour les anglophones, mais $\mathrm{s}=25,58$ et 14,14 respectivement) ${ }^{(61)}$. 
Une sixième variable se rapporte à la lecture des quotidiens et des magazines sur Internet. Les répondants encerclent une réponse sur l'échelle de Likert. Seul le niveau d'instruction joue ici quelque rôle ${ }^{(62)}$ : en deçà du postsecondaire, la moyenne est de 1,44, au delà, de 1,99. Bien que les plus instruits témoignent d'un usage un peu plus fréquent que les autres, on peut constater que, dans les deux cas, les moyennes sont faibles, ce qui indique bien qu'il s'agit d'une activité plutôt rare.

Une septième variable porte sur le clavardage, le chat, et sur la participation aux forums de discussion. À nouveau, les réponses se situent sur une échelle à cinq niveaux. Cette fois-ci, le seul facteur déterminant est l'âge(63). La tendance est nette: plus l'âge du groupe est élevé, moins on incline à s'adonner à cette activité. Les moyennes sont de 1,19 pour les plus vieux, de 1,37 pour les personnes qui ont entre 36 et 50 ans, de 1,77 pour celles qui se situent entre 26 et 35 ans, et de 2,35 pour celles qui ont entre 15 et 25 ans. Il s'agit, une fois encore, d'une activité peu fréquente, comme le démontre la faiblesse des moyennes.

Les deux dernières variables sont des énoncés généraux pour lesquels le répondant doit prendre position sur une échelle à cinq niveaux, « 1 » signifiant «jamais» et « 5 », « souvent». Les énoncés sont les suivants: «J'utilise Internet pour communiquer avec d'autres» et "J'utilise Internet pour trouver des informations". L'usage à des fins communicationnelles ne dépend pas de la langue ${ }^{(64)}$; il est toutefois influencé par le niveau d'instruction ${ }^{(65)}$ et par l'âge ${ }^{(66)}$. Pour les plus instruits, le score est de 3,42 , pour les moins instruits, il est de 2,39; pour les plus jeunes, la moyenne atteint 4,11 , elle décroît ensuite à 3,08 (chez les individus qui ont de 26 à 35 ans), puis à 2,68 (chez ceux qui ont de 36 à 50 ans) et jusqu'à 1,96 (chez les plus vieux). L'usage à des fins informationnelles dépend aussi bien de l'âge $^{(67)}$ et de l'instruction ${ }^{(68)}$ que de la langue ${ }^{(69)}$. Il est moins récurrent avec l'augmentation de l'âge, la 
moyenne passant de 4,06, chez les plus jeunes, à 2,35, chez les plus vieux. Il est moins habituel chez les moins instruits: 2,63 pour 3,71 . Il est moins courant chez les francophones: 3,14 pour 3,99 .

\section{INDIFFÉRENCIATION ET DIFFÉRENCIATION}

Les chiffres relatifs au fait d'avoir chez soi des médias ne signalent d'écart important entre les francophones et les anglophones que pour ce qui est de l'ordinateur, l'appareil meublant plus communément les foyers anglophones. Et cet écart est d'autant plus appréciable qu'il semble structurel; non seulement, en effet, se révèle-t-il si l'on examine les populations dans leur ensemble, mais encore il persiste dans une comparaison qui ne prend en considération que les plus jeunes sur le marché du travail. L'écart est toujours supérieur à $20 \%$. Il témoigne d'une distinction réelle entre les deux groupes linguistiques. Toutefois, cette distinction n'est pas absolue: les chiffres favorisent indubitablement les domiciles anglophones, mais ils indiquent également que pas moins de $60 \%$ des foyers francophones en sont munis.

Pour ce qui est des autres médias - ou services médiatiques - la différence entre les deux groupes linguistiques est rarement supérieure à $10 \%$. Dans les foyers des plus jeunes, pour la plupart des médias, la différence s'estompe. Le lecteur DVD peut retenir l'attention. On l'observe plus fréquemment dans les foyers anglophones, aussi bien pour les échantillons intégraux que pour les échantillons de plus jeunes. Mais on peut supposer que cette technologie est moins rébarbative aux propriétaires d'ordinateurs qu'aux autres, ce que démontre, par ailleurs, une analyse où il apparaît que $88,1 \%$ des foyers dans lesquels on trouve un ordinateur sont aussi munis d'un lecteur DVD ${ }^{(70)}$.

Il semble donc que, d'une façon générale, le domicile de l'anglophone ressemble beaucoup à celui du francophone, 
pour ce qui est de l'équipement médiatique, mais qu'il faille souligner une différence en ce qui a trait à l'ordinateur.

La question se pose alors de savoir comment ces similitudes et cette distinction se traduisent dans les comportements, ou encore de découvrir si les comportements peuvent varier selon d'autres facteurs comme l'âge et le niveau de scolarité. Or, les analyses démontrent effectivement très peu de différences selon la langue: aucune différence significative pour ce qui est de regarder la télévision, de communiquer par téléphone, d'écouter la radio, d'écouter de la musique enregistrée, d'écrire ou de recevoir des lettres en format traditionnel; aucune différence même pour le temps qu'on accorde à l'ordinateur, pour le recours au courriel, pour la lecture sur Internet ou pour le clavardage. On a affaire ici à un net phénomène d'homogénéisation des pratiques, de fusionnement des attitudes culturelles. Mais cette similarité ne repose pas toujours sur les mêmes explications et, en outre, elle connaît des exceptions. Ces explications ne sont pas toujours semblables en ce que souvent on peut évoquer effectivement des moyennes qui sont proches l'une de l'autre et que, d'autres fois, il faut faire état de la prééminence des variations entre les individus sur la différence entre les groupes linguistiques, comme c'est le cas notamment pour le temps que l'on consacre à l'ordinateur. La postmodernité fait ici en sorte que les groupes linguistiques deviennent comparables non pas en ce que les moyennes sont semblables d'un groupe à l'autre, mais bien plutôt, paradoxalement, en ce qu'elle particularise les comportements en transcendant les groupes linguistiques. Les anglophones utilisent en moyenne davantage l'ordinateur que les francophones, comme aurait pu le laisser prévoir le fait qu'ils sont, en proportion, plus nombreux à en posséder un; mais l'incidence de cet usage plus commun pour un groupe linguistique est éliminée par la pluralité des formes particulières qui peuvent être développées. Elle connaît des exceptions dans la logique 
inverse, en ce qu'il y a en réalité quelques cas d'inégalité de moyennes, pour la lecture des quotidiens ou pour celle des revues et des magazines, par exemple.

Cette uniformité étendue et, à la fois, cette importance relative des variations individuelles montrent bien qu'on a sous les yeux des communautés postmodernes qui en sont à des niveaux de richesse comparables. Ces deux communautés se distinguent difficilement l'une de l'autre dans leur rapport aux médias. Des facteurs autres que la langue parviennent davantage à discriminer les comportements et ils le font en dépassant la logique des groupes linguistiques. L'influence de la langue ou de l'instruction est nulle pour certains usages médiatiques, comme pour ceux qui ont trait au temps réservé à la télévision, au nombre de communications téléphoniques ou au temps d'écoute de la radio; mais elle est manifeste pour plusieurs autres: on doit retenir que l'informatique appartient avant tout aux jeunes, même si, en termes de temps, les personnes qui ont de 36 à 50 ans ne sont pas très loin derrière celles qui ont de 15 à 25 ans; on doit aussi remarquer que la lecture est privilégiée par les plus vieux, même s'ils vont moins souvent à la bibliothèque que les plus jeunes, que la musique enregistrée attire plutôt les jeunes; de même, on doit noter que les activités liées à l'informatique, au courrier traditionnel ou à la lecture sont plus fréquentes chez les plus instruits. La postmodernité - comme, à une autre échelle, les sociétés traditionnelles par ailleurs - uniformise alors en produisant des comportements qui sont les mêmes pour des groupes d'âge ou des niveaux d'instruction particuliers, indépendamment de la collectivité dans laquelle on les observe. Mais elle déconstruit en même temps cette homogénéisation, paradoxalement, en faisant que les individus d'autres groupes d'âge, d'autres niveaux de scolarité puissent s'adonner aux activités médiatiques qui sont plus 
propres à un ensemble donné; ainsi, elle réduit l'homogénéisation en étendant le phénomène à d'autres groupes quoiqu'en en réduisant là la fréquence; elle produit donc simultanément la différenciation et l'indifférenciation.

\section{Conclusion}

Les analyses effectuées révèlent qu'on ne peut parler de discrimination des francophones par rapport aux anglophones pour ce qui est de disposer dans son foyer des médias ou d'en faire usage, sinon, et à un certain niveau seulement, en ce qui a trait à l'informatique. Elles témoignent également du fait que les collectivités francoontarienne et anglo-ontarienne sont toutes deux inscrites dans une double évolution, à savoir dans un processus simultané d'homogénéisation et d'hétérogénéisation. Mais les analyses effectuées ici ont des limites: elles portent sur la possession et sur la fréquence des usages; elles ne portent pas sur les contenus, sur les messages qui sont produits, sur ceux qui sont consommés; elles portent sur des fréquences de comportements qui correspondent à des agissements culturels, elles n'ont pas pour objet des symboliques. Maintenant que cette similitude relativement étendue entre les francophones et les anglophones a été établie pour ce qui est des médias qu'on a chez soi et de la fréquence des usages de ces médias, il importe de découvrir à quoi elle correspond au plan symbolique. Et l'on peut se demander si cette équité sert ou dessert la culture minoritaire, si la tendance à l'homogénéisation nuit ou non à la culture franco-ontarienne, aussi bien pour ce qui est des informations qu'elle produit que pour celles auxquelles elle s'expose. 


\section{Notes STATISTIQUeS}

Chacun des tests mesure la probabilité que le lien entre les variables soit attribuable au hasard. Si cette probabilité est inférieure à $5 \%(\mathrm{p}<0,05)$, il est alors entendu que les variations entre les valeurs des variables ne sont pas attribuables au hasard et que, donc, les variables sont liées entre elles. Cette probabilité est mesurée en fonction de différentes distributions aléatoires. On trouvera dans ce texte les distributions $\chi^{2}, t$ et $F$. Plus les valeurs de ces distributions sont élevées, plus est fort le lien entre les variables et plus est faible la probabilité que le lien entre les variables soit attribuable au hasard. La statistique $\chi^{2}$ s'emploie pour mesurer le lien entre deux variables nominales, par exemple le sexe et le fait qu'on réponde oui ou non. La statistique $t$ sert à vérifier s'il y a un lien significatif ou inférable entre deux moyennes, par exemple entre les hommes et les femmes pour le temps qui est consacré à la télévision. La variable aléatoire F, enfin, permet de vérifier si la différence entre plus de deux groupes (par exemple trois groupes d'âges) ou si les variations en fonction de plus d'une variable nominale ou nominalisée (par exemple le sexe, d'une part, et les groupes d'âge, d'autre part) sont attribuables au hasard. 


$$
\begin{aligned}
& { }^{1} \chi_{\text {(corrige) }}^{2}=3,15 ; \mathrm{p}=0,08 . \\
& { }^{2} \mathrm{t}_{(949)}=11,96 ; \mathrm{p}<0,001 \text {. } \\
& { }^{3} \chi_{\text {(corrige) }}^{2}=0,96 ; p \geq 0,05 \text {. } \\
& { }^{4} \chi_{\text {(corrige) }}^{2}=0,13 ; p \geq 0,05 \text {. } \\
& { }^{5} \chi_{\text {(corige) }}^{2}=0,80 ; p \geq 0,05 \text {. } \\
& { }^{6} \chi_{\text {(corrige) }}^{2}=0,09 ; \mathrm{p} \geq 0,05 \text {. } \\
& { }^{7} \chi_{\text {(corrige) }}^{2}=2,04 ; \mathrm{p} \geq 0,05 \text {. } \\
& { }^{8} \chi_{\text {(corrigé) }}^{2}=2,43 ; \mathrm{p} \geq 0,05 \text {. } \\
& { }^{9} \chi_{\text {(corrigé) }}^{2}=14,95 ; p<0,05 \text {. } \\
& { }^{10} \chi_{\text {(corrige) }}^{2}=32,52 ; \mathrm{p}<0,05 \text {. } \\
& { }^{11} \chi_{\text {(corrige) }}^{2}=9,91 ; p<0,05 \text {. } \\
& { }^{12} \chi_{\text {(corrige) }}^{2}=21,83 ; \mathrm{p}<0,05 \text {. } \\
& { }^{13} \chi_{\text {(corrige) }}^{2}=5,21 ; \mathrm{p}<0,05 \text {. } \\
& { }^{14} \chi_{\text {(corrige) }}^{2}=29,34 ; p<0,05 \text {. } \\
& { }^{15} \chi_{\text {(corrigé) }}^{2}=39,20 ; p<0,05 \text {. } \\
& { }^{16} \chi_{\text {(corrige) }}^{2}=38,53 ; \mathrm{p}<0,05 \text {. } \\
& { }^{17} \chi_{\text {(corrige) }}^{2}=44,57 ; p<0,05 \text {. } \\
& { }^{18} \chi_{\text {(corrige) }}^{2}=10,21 ; p<0,05 \text {. } \\
& { }^{19} \chi_{\text {(corrige) }}^{2}=5,47 ; \mathrm{p}<0,05 \text {. } \\
& { }^{20} \chi_{\text {(corrige) }}^{2}=14,18 ; p<0,05 \text {. } \\
& { }^{21} \chi_{\text {(corrige) }}^{2}=6,65 ; \mathrm{p}<0,05 \text {. } \\
& { }^{22} \chi_{\text {(corrige) }}^{2}=8,31 ; \mathrm{p}<0,05 \text {. } \\
& { }^{23} \chi_{\text {(corrige) }}^{2}=4,62 ; \mathrm{p}<0,05 \text {. } \\
& { }^{24} \chi_{\text {(corrige) }}^{2}=5,90 ; p<0,05 \text {. } \\
& { }^{25} \chi_{\text {(corrige) }}^{2}=8,57 ; \mathrm{p}<0,05 \text {. } \\
& { }^{26} \chi_{\text {(corrige) }}^{2}=3,97 ; \mathrm{p}<0,05 \text {. } \\
& { }^{27} \chi_{\text {(corrige) }}^{2}=8,38 ; \mathrm{p}<0,05 \text {. } \\
& { }^{28} \chi_{\text {(corrige) }}^{2}=0,03 ; p \geq 0,05 \text {. } \\
& { }^{29} \chi_{\text {(corrige) }}^{2}=0,02 ; \mathrm{p} \geq 0,05 \text {. } \\
& { }^{30} \chi_{\text {(corrige) }}^{2}=0,49 ; p \geq 0,05 \text {. } \\
& { }^{31} \chi_{\text {(corrige) }}^{2}=0,01 ; p \geq 0,05 \text {. } \\
& { }^{32} \mathrm{~F}_{(1 ; 551)}=0,30 ; \mathrm{p}=0,59 \text {. } \\
& { }^{33} \mathrm{t}_{(372,57)}=-1,05 ; \mathrm{p} \geq 0,05 \text {. } \\
& { }^{34} t_{(212)}=-1,47 ; p \geq 0,05 \text {. } \\
& { }^{35} \mathrm{t}_{(62,70)}=-1,40 ; \mathrm{p} \geq 0,05 \text {. } \\
& { }^{36} \mathrm{t}_{(287,25)}=-1,82 ; \mathrm{p} \geq 0,05 \text {. } \\
& { }^{37} \mathrm{~F}_{(3 ; 923)}=4,73 ; \mathrm{p}<0,01 \text {. } \\
& { }^{38} \mathrm{~F}_{(1: 916)}=0,95 ; \mathrm{p}=0,33 \text {. } \\
& { }^{39} \mathrm{~F}_{(3 ; 910)}=23,29 ; \mathrm{p}<0,001 \text {. } \\
& { }^{40} \mathrm{~F}_{(1 ; 915)}=1,90 ; \mathrm{p}=0,30 \text {. } \\
& { }^{41} \mathrm{~F}_{(3 ; 915)}=16,09 ; \mathrm{p}<0,001 \text {. } \\
& { }^{42} \mathrm{~F}_{(1 ; 915)}=7,30 ; \mathrm{p}<0,01 \text {. } \\
& { }^{43} \mathrm{~F}_{(3 ; 921)}=37,42 ; \mathrm{p}<0,001 \text {. }
\end{aligned}
$$




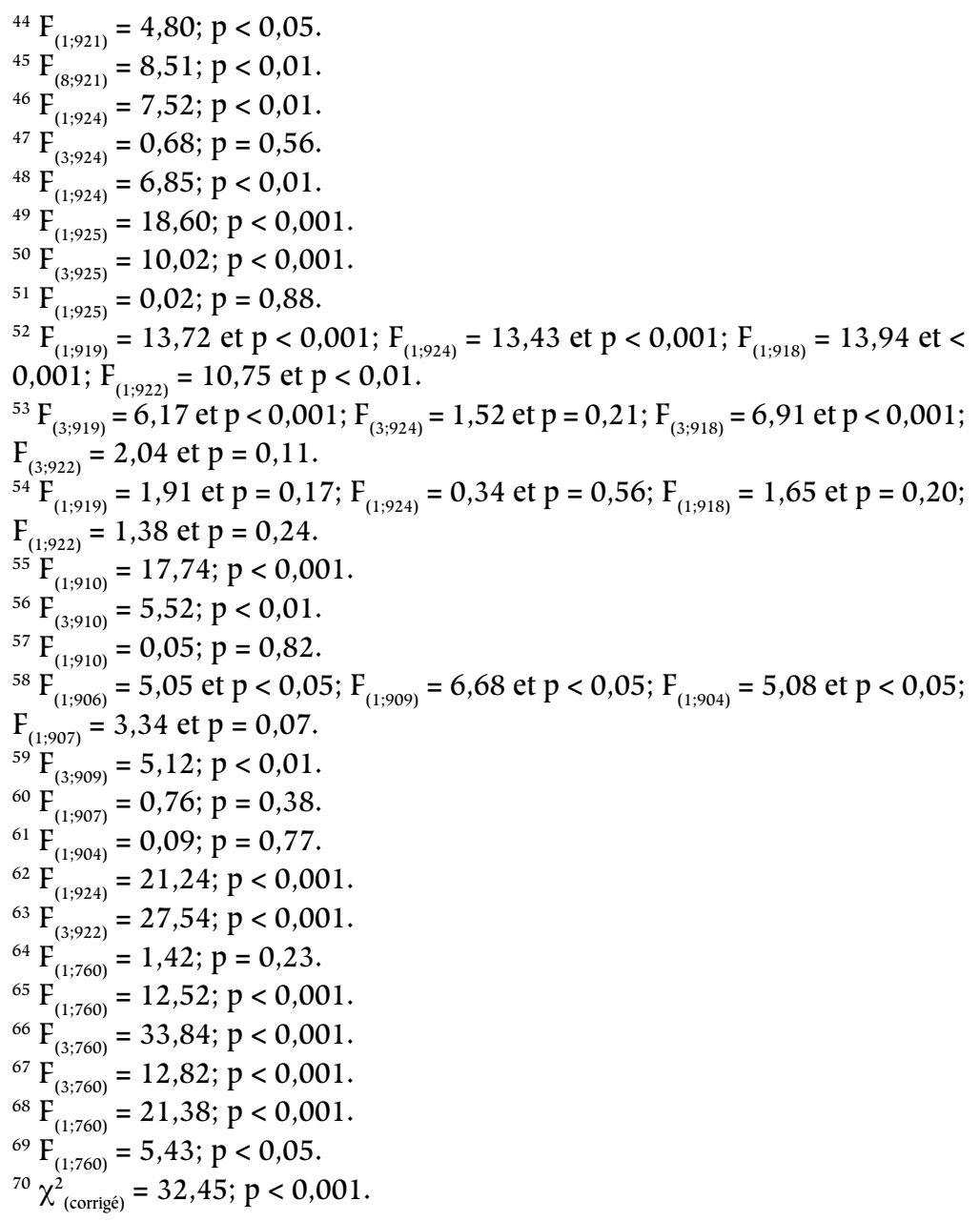




\section{QUESTIONNAIRE SUR L'USAGE DES MÉDIAS}

Le questionnaire que vous avez entre les mains s'adresse à tout adulte. Les questions qu'il comporte permettront d'obtenir des informations sur l'usage des médias. On vous demande de répondre aux questions avec sincérité et au meilleur de votre connaissance. Il ne s'agit pas d'un test. Bien entendu, vous n'êtes pas obligé-e de répondre au questionnaire ni, si vous y répondez, de répondre aux questions qui pourraient vous déplaire. Le succès de cette recherche, toutefois, dépend de votre collaboration.

Les responsables de la recherche vous assurent de la confidentialité de toutes vos réponses. En aucun endroit on ne vous demande votre nom. Les données ne seront publiées qu'en tant qu'elles seront regroupées, ce qui rendra impossible l'identification de cas particuliers.

$\mathrm{D}^{\mathrm{re}}$ Monique Benoit

et $\mathrm{D}^{\mathrm{r}}$ Simon Laflamme

$\mathrm{D}^{\mathrm{re}}$ Judith Lazar

Département de sociologie

CURAPP-Centre universitaire

Université Laurentienne

Chemin du Lac Ramsey

de recherches administratives

et politiques de Picardie

Sudbury (Ontario)

B.P. 2716

P3E 2C6

80027 Amiens Cedex 1

téléphone: (705) 675-1151, poste 4217

téléphone: 01-45-89-26-72 
1. Quel âge avez-vous? ans

2. Quel est votre sexe?

[Encerclez le chiffre qui correspond à la bonne réponse.]

féminin 1

masculin 2

3. Quel est votre statut matrimonial?

[Encerclez le chiffre qui correspond le mieux à votre situation.]

célibataire

séparé-e ou divorcé-e

marié-e ou conjoint-e de fait

veuf-ve

autre
1 [Je n'ai jamais vécu en couple.]

2 Depuis combien de temps n'êtes-vous plus en couple? ans

3 Depuis combien de temps vivez-vous en couple? ans

4 Depuis combien de temps n'êtes-vous plus en couple(e)? ans

5 Précisez:

4. Y a-t-il des enfants dans votre foyer?

[Encerclez le chiffre qui correspond à la bonne réponse.]

$\begin{array}{ll}\text { oui } & 1 \\ \text { non } & 2\end{array}$

5. Vivez-vous seul-e?

[Encerclez le chiffre qui correspond à la bonne réponse.]

$\begin{array}{ll}\text { oui } & 1 \\ \text { non } & 2\end{array}$

6. Vivez-vous en milieu urbain ou en milieu rural?

[Encerclez le chiffre qui correspond à la bonne réponse.]

urbain 1

rural 2

7. Indiquez le niveau d'éducation le plus élevé que vous ayez atteint. [Encerclez le chiffre qui correspond à la bonne réponse.]

quelques années de l'école élémentaire .............. 1

cours élémentaire terminé .................................... 2

quelques années de l'école secondaire ............... 3

diplôme d'études secondaires ............................... 4

quelques cours de niveau collégial ..................... 5

diplôme d'études collégiales ................................ 6

quelques années de niveau universitaire ............ 7

diplôme d'études de premier cycle ........................ 8

diplôme d'études supérieures .............................. 9 
8. Quelle est votre occupation?

[Encerclez le chiffre qui correspond à la réponse qui traduit le mieux votre situation.]

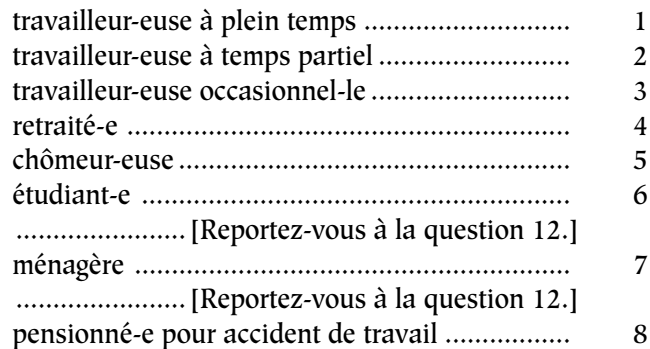

9. Quel travail exercez-vous (ou exerciez-vous avant la retraite ou le chômage)? [Soyez spécifique. Par exemple: bûcheron à son propre compte; enseignante au secondaire; ou vendeuse dans un grand magasin.]

Si vous n'êtes pas à l'emploi pour le moment ou si vous ne travaillez pas contre rémunération, reportez-vous à la question 12 .

10. Combien d'heures par semaine travaillez-vous normalement?

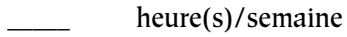

11. Quelle est votre situation relativement à votre lieu de travail? [Encerclez le chiffre qui correspond à la réponse qui traduit le mieux votre situation.]

je travaille principalement à l'extérieur du foyer 1 je travaille principalement au foyer 2 
12. Dans votre domicile, y a-t-il un (ou plusieurs) téléviseur(s)? [Encerclez le chiffre qui correspond à la bonne réponse.]

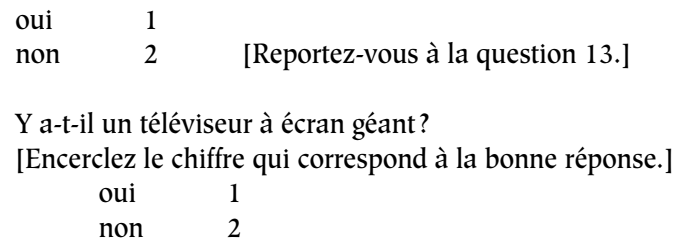

Le téléviseur est-il branché au service du câble ou à une antenne parabolique?

[Encerclez le chiffre qui correspond à la bonne réponse.]

$$
\begin{array}{ll}
\text { oui } & 1 \\
\text { non } & 2
\end{array}
$$

13. Dans votre domicile, y a-t-il un magnétoscope?

[Encerclez le chiffre qui correspond à la bonne réponse.]

$\begin{array}{ll}\text { oui } & 1 \\ \text { non } & 2\end{array}$

14. Dans votre domicile, y a-t-il un lecteur DVD?

[Encerclez le chiffre qui correspond à la bonne réponse.]

$$
\begin{array}{ll}
\text { oui } & 1 \\
\text { non } & 2
\end{array}
$$

15. Dans votre domicile, y a-t-il un (ou plusieurs) appareil(s) radio(s)? [Encerclez le chiffre qui correspond à la bonne réponse.]

$$
\begin{array}{ll}
\text { oui } & 1 \\
\text { non } & 2
\end{array}
$$

16. Dans votre domicile, y a-t-il un lecteur de disques compacts? [Encerclez le chiffre qui correspond à la bonne réponse.]

$\begin{array}{ll}\text { oui } & 1 \\ \text { non } & 2\end{array}$

17. Dans votre domicile, y a-t-il un télécopieur (fax)?

[Encerclez le chiffre qui correspond à la bonne réponse.]

$\begin{array}{ccc}\text { oui } & 1 & \text { Sinon, disposez-vous d'un télécopieur? } \\ \text { non } & 2 & \begin{array}{c}\text { [Encerclez le chiffre qui correspond à la } \\ \text { bonne réponse.] } \\ \text { oui } \\ \text { non }\end{array} \\ & & 1\end{array}$


18. Dans votre domicile, y a-t-il une ligne téléphonique?

[Encerclez le chiffre qui correspond à la bonne réponse.]

oui 1

non $\quad 2 \quad$ [Reportez-vous à la question 19.]

Est-ce qu'au moins un des appareils est portable?

[Encerclez le chiffre qui correspond à la bonne réponse.]

oui 1

non 2

Ce service téléphonique est-il branché à un répondeur?

[Encerclez le chiffre qui correspond à la bonne réponse.]

oui 1

non 2

Ce service téléphonique comporte-t-il un afficheur?

[Encerclez le chiffre qui correspond à la bonne réponse.]

oui 1

non 2

19. Disposez-vous d'un téléphone cellulaire?

[Encerclez le chiffre qui correspond à la bonne réponse.]

oui 1

non $\quad 2 \quad$ [Reportez-vous à la question 20.]

Ce service téléphonique est-il branché à un répondeur?

[Encerclez le chiffre qui correspond à la bonne réponse.]

oui 1

non 2

20. Dans votre domicile, y a-t-il un ordinateur?

[Encerclez le chiffre qui correspond à la bonne réponse.]

oui 1

non 2 [Reportez-vous à la question 21.]

Dans votre domicile, y a-t-il un branchement Internet?

[Encerclez le chiffre qui correspond à la bonne réponse.]

oui 1

non 2

Dans votre domicile, avez-vous accès à un service de courrier

électronique?

[Encerclez le chiffre qui correspond à la bonne réponse.]

oui 1

non 2

Dans votre domicile, y a-t-il un scanner?

[Encerclez le chiffre qui correspond à la bonne réponse.]

oui 1

non 2 
21. Répondez à chacune des questions suivantes qui se rapportent à des activités spécifiques.

[Inscrivez le chiffre dans l'espace aménagé à cet effet. Si vous ne disposez pas de l'équipement ou si l'activité en question correspond à une fréquence nulle, inscrivez simplement le chiffre zéro (0).]

De façon approximative, combien de films visionnez-vous par mois à partir d'une cassette vidéo ou d'un disque DVD?

De façon approximative, combien de télécopies (fax) recevez-vous ou envoyez-vous par mois dans le cadre de votre travail? De façon approximative, combien de télécopies (fax) recevez-vous ou envoyez-vous par mois en dehors du cadre de votre travail? De façon approximative, combien d'appels téléphoniques locaux faites-vous ou recevez-vous chaque semaine dans le cadre de votre travail?

De façon approximative, combien d'appels téléphoniques interurbains faites-vous ou recevez-vous chaque semaine dans le cadre de votre travail? De façon approximative, combien d'appels téléphoniques locaux faites-vous ou recevez-vous chaque semaine en dehors du cadre de votre travail? De façon approximative, combien d'appels téléphoniques interurbains faites-vous ou recevez-vous chaque semaine en dehors du cadre de votre travail? De façon approximative, combien de messages électroniques recevez-vous chaque semaine dans le cadre de votre travail?

De façon approximative, combien de messages électroniques recevez-vous chaque semaine en dehors du cadre de votre travail? par mois

De façon approximative, combien de messages électroniques écrivez-vous chaque semaine dans le cadre de votre travail? par semaine De façon approximative, combien de messages électroniques écrivez-vous chaque semaine en dehors du cadre de votre travail? par semaine par semaine par semaine

Si vous n'avez pas accès à un service Internet, reportez vous à la question 24 . 
22. Énumérez, par ordre de priorité, les cinq principaux usages que vous faites de l'Internet.

1.

2.

3.

4.

5.

23. Indiquez, pour chacune des activités suivantes, l'usage qui correspond le mieux à votre situation.

[Le chiffre « 1 » signifie que vous ne vous adonnez jamais à l'activité qui est désignée par l'énoncé; le chiffre « 5 » signifie que vous vous y adonnez souvent; les autres chiffres correspondent à des positions intermédiaires.]

J'utilise Internet pour communiquer

\begin{tabular}{lllllr}
\multicolumn{2}{c}{ jamais } & & \multicolumn{2}{r}{ souvent } \\
& & & & \\
1 & 2 & 3 & 4 & 5 \\
1 & 2 & 3 & 4 & 5
\end{tabular}

24. Indiquez, pour chacune des activités suivantes, l'usage qui correspond le mieux à votre situation.

[Le chiffre « 1 " signifie que vous ne vous adonnez jamais à l'activité qui est désignée par l'énoncé; le chiffre « 5 » signifie que vous vous y adonnez souvent; les autres chiffres correspondent à des positions intermédiaires.]

Je lis des quotidiens imprimés

Je lis des revues ou des magazines imprimés jamais souvent Je lis des quotidiens ou des magazines sur Internet J'écris des lettres dans le cadre de mon travail J'écris des lettres en dehors du cadre de mon travail Je reçois des lettres dans le cadre de mon travail 1 Je reçois des lettres en dehors du cadre de mon travail Je reçois des parents ou des amis à la maison Je vais à la bibliothèque

Je vais au restaurant

Je vais au théâtre

Je vais au cinéma

Je «chat» ou j'interviens dans le cadre de forums sur Internet

$\begin{array}{lllll}1 & 2 & 3 & 4 & 5\end{array}$

$\begin{array}{lllll}1 & 2 & 3 & 4 & 5\end{array}$

$\begin{array}{lllll}1 & 2 & 3 & 4 & 5\end{array}$

$\begin{array}{lllll}1 & 2 & 3 & 4 & 5\end{array}$

$\begin{array}{lllll}1 & 2 & 3 & 4 & 5\end{array}$

$\begin{array}{lllll}1 & 2 & 3 & 4 & 5\end{array}$

$\begin{array}{lllll}1 & 2 & 3 & 4 & 5\end{array}$

$\begin{array}{lllll}1 & 2 & 3 & 4 & 5\end{array}$

$\begin{array}{lllll}1 & 2 & 3 & 4 & 5\end{array}$

$\begin{array}{lllll}1 & 2 & 3 & 4 & 5\end{array}$

$\begin{array}{lllll}1 & 2 & 3 & 4 & 5\end{array}$

$\begin{array}{lllll}1 & 2 & 3 & 4 & 5\end{array}$

Je réponds à des petites annonces de rencontre ou je recours à des services de rencontre dans des journaux ou sur Internet 
25. À combien estimeriez-vous le nombre de sorties (restaurant, cinéma, discothèque, musée, visites chez des amis...) que vous faites chaque mois? sorties par mois.

26. Recevez-vous des journaux ou des magazines à la maison? [Encerclez le chiffre qui correspond à la bonne réponse.]

$\begin{array}{ll}\text { oui } & 1 \\ \text { non } & 2\end{array}$

27. Combien d'heures par semaine consacrez-vous normalement aux activités suivantes?

[Inscrivez le nombre d'heures dans l'espace aménagé à cet effet.]

Je regarde la télévision (visionnement de vidéos y compris)

J'écoute la radio heure(s)/semaine

Je fais des lectures en dehors du cadre de mes études ou de mon travail

J'écoute de la musique enregistrée heure(s)/semaine

Je parle au téléphone en dehors du cadre de mon travail heure(s)/semaine Je suis devant un écran d'ordinateur heure(s)/semaine heure(s)/semaine

\section{MERCI DE VOTRE COLLABORATION}

